\section{(6) OPEN ACCESS}

\title{
Translating knowledge from Pakistan's second generation surveillance system to other global contexts
}

\author{
Alix Adrien, ${ }^{1,2}$ Laura H Thompson, ${ }^{3}$ Chris P Archibald, ${ }^{4}$ Paul A Sandstrom, ${ }^{4}$ \\ Michelle Munro, ${ }^{5,6}$ Faran Emmanuel, $^{3,6}$ James F Blanchard $^{3}$
}

\begin{abstract}
${ }^{1}$ Direction de Santé Publique, Agence de la Santé et des Services Sociaux de Montreal, Montréal, Quebec, Canada ${ }^{2}$ Department of Epidemiology, Biostatistics and Occupational Health, McGill University, Montreal, Quebec, Canada ${ }^{3}$ Department of Community Health Sciences, Centre for Global Public Health, University of Manitoba, Winnipeg, Manitoba, Canada

${ }^{4}$ National HIV and Retrovirology Laboratories, Public Health Agency of Canada, Ottawa, Ontario, Canada

${ }^{5}$ Agriteam Canada Consulting Ltd, Gatineau, Quebec, Canada

${ }^{6}$ Canada-Pakistan HIVIAIDS

Surveillance Project, Islamabad, Pakistan
\end{abstract}

\section{Correspondence to}

Dr James F. Blanchard, Centre

for Global Public Health,

Department of Community

Health Sciences, University of

Manitoba, R070-771

McDermot Avenue, Winnipeg, MB R3E 0T6;

james_blanchard@umanitoba. ca

Received 8 November 2012 Revised 8 November 2012 Accepted 15 November 2012 Published Online First 20 December 2012

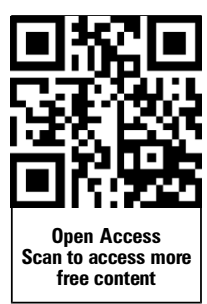

To cite: Adrien $A$, Thompson LH, Archibald CP et al. Sex Transm Infect 2013;89:ii53-ii57.

\section{ABSTRACT}

Background From 2004 to 2011, a collaborative project was undertaken to enhance the capacity of the Government of Pakistan to implement an effective second-generation surveillance system for HIVIAIDS, known as the HIVIAIDS Surveillance Project (HASP). In four separate rounds, behavioural questionnaires were administered among injection drug users, and female, male and hijra (transgender) sex workers. Dried blood spots were collected for HIV testing.

Methods Through interviews with project staff in Pakistan and Canada, we have undertaken a critical review of the role of HASP in generating, using and translating knowledge, with an emphasis on capacity building within both the donor and recipient countries. We also documented ongoing and future opportunities for the translation of knowledge produced through HASP.

Results Knowledge translation activities have included educational workshops and consultations held in places as diverse as Colombia and Cairo, and the implementation of HASP methodologies in Asia, the Middle East and sub-Saharan Africa. HASP methodologies have been incorporated in multiple WHO reports. Importantly, the donor country, Canada, has benefited in significant ways from this partnership. Operational and logistical lessons from HASP have, in turn, improved how surveillance is performed in Canada. Through this project, significant capacity was built among the staff of HASP, non-governmental organisations which were engaged as implementation partners, data coordination units which were established in each province, and in the laboratory. As is to be expected, different organisations have different agendas and priorities, requiring negotiation, at times, to ensure the success of collaborative activities. Overall, there has been considerable interest in and opportunities made for learning about the methodologies and approaches employed by HASP.

Conclusions Generally, the recognition of the strengths of the approaches and methodologies used by HASP has ensured an appetite for opportunities of mutual learning.

\section{INTRODUCTION}

From 2004 to 2011, a collaborative project was undertaken to enhance the capacity of the Government of Pakistan to implement an effective second-generation surveillance (SGS) system for HIV/AIDS, known as the Canada-Pakistan HIV/
AIDS Surveillance Project (HASP). SGS is a comprehensive process of systematically collecting, analysing and interpreting behavioural and biological data for the purpose of tracking changes in an epidemic over time, which may be used to inform the development, implementation and evaluation of HIV prevention programmes. ${ }^{1}$

The particular data collected by SGS may be determined by the local epidemic typology. Three broad epidemic typologies have been advanced: concentrated, generalised and mixed. ${ }^{2}$ In concentrated epidemics, HIV transmission is driven by networks of key populations at greater risk of HIV infection such as male sex workers (MSWs) and female sex workers (FSWs) and their clients, injection drug users (IDUs) and their sexual partners, and men who have sex with men (MSM) who have relatively high numbers of sexual partners. Pakistan's HIV epidemic is classified as a concentrated epidemic, with the majority of transmission occurring among IDUs, and recent evidence of transmission occurring among MSWs, FSWs and 'hijra' (transgender) sex workers. ${ }^{3}$ In this context, SGS focuses on collecting information related to HIV transmission among these subpopulations.

A wealth of knowledge and expertise has been built over 8 years of supporting Pakistan to implement SGS, including knowledge about resource, infrastructure and supply needs, sampling methodologies, the design and administration of surveys, the logistics of collecting and processing biological specimens on a large scale, the development and management of databases, and knowledge dissemination. The lessons learned have provided valuable insights which have informed methodologies used for surveillance projects in Canada and elsewhere as part of the knowledge translation component of the project.

Knowledge translation provides opportunities for experiences and lessons gained over the course of a project to contribute to collective bodies of knowledge, enhancing the ability for similar projects to be successfully implemented. In the context of public health, knowledge translation is a dynamic and iterative process that involves the compilation, exchange and ethical application of knowledge to improve the effectiveness and efficiency of health services and systems. ${ }^{4}$ In global public health, joint initiatives between different countries provide opportunities for enhanced learning, as the application of methodologies and techniques in different contexts may bring 
unexpected strengths and weaknesses to light and reveal new opportunities. In 1990, the report Health Research: Essential Link to Equity in Development was published, which emphasised the importance of every country having the ability to pursue research and contribute to the international advancement of knowledge through partnerships with researchers in other countries. ${ }^{5}$ As Frenk notes, this was the first major challenge to the reigning paradigm of unidirectional problem solving, whereby solutions were 'exported from the north to the south' ${ }^{6}$ Indeed, most publications related to knowledge translation in global public health focus on barriers to the adoption of knowledge and the need to strengthen the health systems of developing countries. ${ }^{7-9}$ This paper will instead provide an overview of the role of HASP in generating, using and translating knowledge, with an emphasis on capacity building within both the donor and recipient countries, and will outline ongoing and future opportunities for the translation of knowledge produced through HASP.

\section{METHODS}

Through interviews with project staff in Pakistan and Canada in 2011, we have undertaken a critical review of the role of HASP in generating, using and translating knowledge, with an emphasis on capacity building within both the donor and recipient countries. Data collection and analysis as part of HASP was approved by the institutional ethical review boards of HOPE International (a non-governmental health and research organization; originally Health Oriented Preventive Education) and the Public Health Agency of Canada.

\section{RESULTS}

\section{Description of HASP}

HASP was the first systematic HIV surveillance system in Pakistan. Prior to HASP, HIV surveillance in Pakistan had been largely passive, with disease trends monitored through clinical samples and occasional ad hoc studies. HASP enabled Pakistan to institutionalise true SGS allowing for better understanding of the distribution of and interactions among risk behaviours. At the time, most HIV surveillance systems outside high-income countries had been developed for the generalised epidemics in sub-Saharan Africa or used convenience samples such as antenatal clinic attendees, so there was a need to develop a new framework appropriate for the concentrated epidemic of Pakistan's unique situation. In addition to the unique social and political context of Pakistan and HIV transmission dynamics within the country, the framework was developed to incorporate sampling methodologies and questionnaires appropriate for subpopulations unique to South Asia. For example, hijra sex workers, transgender individuals who perform at ceremonies and practice sex work through large established networks, were included.

To establish the project, staff were assigned to a central data coordinating unit, a national field epidemiologist was placed within National AIDS Control Program (NACP), and surveillance positions were designated at the provincial level to support Government of Pakistan counterparts. Provincial data coordination units were established in each of the provinces for local data entry, to be transferred later to HASP staff for cleaning and analysis. HASP mapping methodologies were developed, piloted, evaluated and standardised as the official, government-approved approach. After pilot testing, four rounds of mapping and surveillance took place among IDUs, MSWs, FSWs and hijra sex workers over a period of 7 years. The numbers of cities varied each round, with certain key cities and key sub-populations included every round to allow for comparable data. The SGS activities included mapping, questionnaire administration and dried blood spot collection.

The first step of HASP methodology (figure 1) is geographic mapping to obtain a better understanding of the size of key populations and patterns of risk behaviour. The approach involves characterising high-risk activities such as sex work and injection drug use, and estimating the number of individuals involved in these activities. In addition, mapping identifies and characterises locations where high-risk activities take place. Finally, mapping provides information about sub-types of highrisk activities (eg street-based sex work and brothel-based sex work). This information is critical for developing sampling strategies that can ensure selection biases are minimised and information collected is representative of the sub-population studied. After many discussions, it was decided that recruitment of key populations should take place through offices dedicated specifically to HASP rather than pre-existing sexually transmitted infection clinics and primary health centres. Non-governmental organisations (NGOs) were engaged as implementation partners in many aspects of surveillance planning and implementation, including mapping, the recruitment of key population members and data collection. Through the use of questionnaires and dried blood spot collection at regular intervals, information about HIV prevalence and key behavioural and structural vulnerability indicators were then obtained from IDUs, MSWs, FSWs and hijra sex workers. Mapping and integrated behavioural and biological surveillance were conducted in four separate rounds and in many cities across Pakistan, allowing for geographic comparisons and the analysis of temporal trends.

To provide a comprehensive understanding of the local HIV epidemic, in addition to HIV prevalence, HASP also performed HIV molecular subtype analysis. At times, qualitative approaches including in-depth interviews and focus groups were used to obtain a better understanding of the risk environment. Through this process, various protocols have been developed, including surveillance methodologies, ethics and gender guidelines, and supervision and monitoring protocols. Data management and reporting systems were developed, with the ability to integrate data from diverse sources to provide a more complete understanding of the epidemic. As a result of training and experience gained through this SGS project, expertise in data management and analysis, surveillance and epidemiology have been built in Pakistan, which now has a more comprehensive and centralised surveillance system than Canada or the USA. Although knowledge use has depended, to some extent, on the availability of funding and on political will to implement relevant programmes, national surveillance reports have been released and used to inform the development of HIV programme plans, donor funding proposals and subsequent disbursements, Joint United Nations Programme on HIV/AIDS (UNAIDS) HIV estimates, and improvements to and scale-up of service delivery and prevention programming. Through this collaborative process, Pakistani counterparts participated in supporting and administering large-scale surveillance, developing evidence-based policy and programmes and improving HIV prevention and care infrastructure in terms of both reach and quality. 


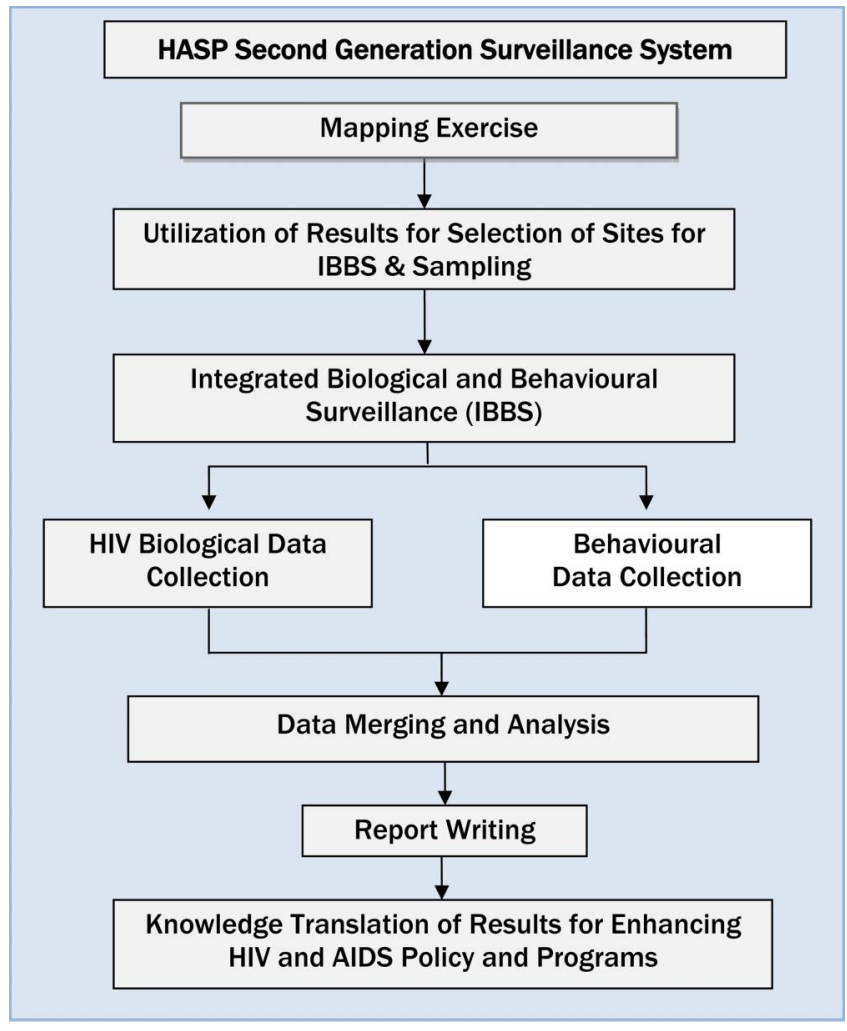

Figure 1 HASP methodology.

\section{Benefits to Pakistan}

- For the first time, data representative of the HIV epidemic among key populations across Pakistan was available to inform HIV prevention policies and programmes;

- The collaborative nature of this effort between an international team of technical experts, technical staff in Pakistan and those in positions within Pakistan to influence policy and programmes ensured that all counterparts would benefit from knowledge about the SGS and development of the SGS;

- The ability to adapt and refine methodologies developed for India to Pakistan and elsewhere;

- Laboratory capacity was built in Pakistan; the process can inform the development of similar systems elsewhere.

- The technical capacity to conduct surveillance and manage data built in provinces and NGOs.

Funded by the Canadian International Development Agency, HASP involved multilateral partnerships between Canadian and Pakistani organisations. The Canadian partners included the University of Manitoba, ProAction: Partners for Community Health, the Public Health Agency of Canada, and Agriteam Canada. The partners in Pakistan included the Ministry of Health, the National AIDS Control Program, provincial AIDS control programs, and a national technical advisory group for HIV surveillance and research within Pakistan.

\section{HASP knowledge translation initiatives}

Knowledge translation initiatives have been used to disseminate knowledge generated in Pakistan over the course of HASP and have informed HASP methodologies from the very beginning. Key HASP staff travelled to India to learn from the large-scale key population mapping and surveillance projects that have taken place in the state of Karnataka over the past decade, informing the design of HIV prevention programmes being delivered with high coverage of target populations, particularly FSWs and MSM. ${ }^{10}$ The ability to adapt methodologies used in India to the context of Pakistan provided confidence in the ability to adapt these methodologies to specific settings. Much learning took place over the course of HASP, allowing the methodologies used in Pakistan to be improved and refined from round to round. Also, it was essential for methodologies to be flexible so that the surveillance system could be responsive to changes in the local context, communities and epidemics.

The use of dried blood spots for HIV testing and molecular typing has been demonstrated to be safe and cost-effective and is now accepted as an important component of a SGS system. Significant laboratory capacity was built in Pakistan through HASP, both in terms of technical ability to test dried blood spots and in the ability to handle large volumes of specimens. In the fourth round of SGS, a new specimen tracking system was developed, allowing the team to reliably monitor the quality of the specimens and to archive the specimens centrally under ideal conditions, while also permitting decentralised serologic testing. This was a significant advance and has the potential to also inform how similar systems can be improved in Canada. WHO guidelines for the use of dried blood spots for HIV surveillance were informed by experiences from HASP. ${ }^{11}$

HASP staff held workshops to train provincial and NGO staff in HASP methodologies and developed their skills in the use of surveillance data for program planning and monitoring.

To allow others to capitalise on the lessons learned over the course of HASP, ongoing knowledge translation has been taking place through discussions, consultations and capacity-building initiatives with countries in the Middle East and North Africa (MENA), Central Asia, subSaharan Africa, Colombia and Canada. Given the societal and religious similarities between Pakistan and countries in the Middle East, methodologies and approaches developed through HASP are particularly applicable to the context of the MENA region. Mapping, venue-based sampling and population size estimation methodologies which were originally developed in Karnataka, India, have been adapted and optimised for difficult-to-reach networks in Pakistan and have since been implemented in other Islamic countries such as Maldives, Afghanistan and Yemen, as well as in Sri Lanka, China, Nigeria and Kenya. These methodologies have been presented at several meetings, including an inter-country consultation on preventing HIV among IDUs held in Kolkata, India (April 2007), a Pan American Health Organization (PAHO) meeting on designing SGS among MSM in Colombia (May 2007) and at the Second Global HIV/AIDS Surveillance Meeting held in Bangkok, Thailand (March 2009). After these presentations, the WHO Eastern Mediterranean Regional Office expressed interest in learning more about this methodology. A regional workshop on size estimation techniques for key populations was conducted by the Regional Knowledge Hub for HIV/AIDS Surveillance in Kerman, Iran, in October 2009 with representatives from over ten countries in attendance. Following this meeting, a mapping fact sheet was prepared for use in this context. ${ }^{12}$ the WHO Eastern Mediterranean Regional Office published guidelines for HIV surveillance in the MENA region which incorporates numerous examples from HASP. ${ }^{13}$ The methodologies were presented again at the MENA Regional Expert Group Meeting on Strategic Information and Surveillance in Cairo in May 2011. Pakistan's SGS was regarded as one of the best in the region and strong interest was expressed in further learning from its successes. 


\section{Benefits to other countries}

- Use of dried blood spots was demonstrated to be safe and cost-effective; now it is standard part of second generation surveillance.

- Methodologies developed in Pakistan are particularly appropriate for use in countries in the MENA region and in other Islamic countries.

- Methodologies developed in Canada were refined in Pakistan at a large scale, improving the confidence and experience in these methods and the knowledge required to be able to implement them at a large scale in Canada.

In 2010, HASP methodologies were used to map and estimate the size of MSM populations in Yemen. Due to the political and security situation in Yemen at the time, the team was not able to complete this study, but initial spot validation for recruitment of MSM for integrated behavioural and biological surveillance was completed and there are plans to continue this work when the situation changes.

Importantly, the donor country, Canada, has benefited in significant ways from this partnership. The Public Health Agency of Canada, one of the project partners, performs mapping, HIV behavioural and biological surveillance among MSM, IDUs and street youth across Canada in collaboration with provincial and local partners. ${ }^{14-16}$ Many of the same techniques used in these Canadian studies were refined and implemented on a very large scale in Pakistan as part of HASP. Subsequently, a number of operational and logistical lessons from HASP have, in turn, improved how surveillance is performed in Canada, especially with respect to scaling up such studies. The lessons learned from HASP have also provided the leads of the Canadian studies with additional confidence and experience in these methods and provided institutional review boards and partners across Canada with increased confidence in the safety, acceptability and effectiveness of these methods.

Donor countries benefit greatly from international capacity building projects. A systematic approach to document and publicise the ways in which donor countries benefit from international capacity building projects would be useful to ensure ongoing support in foreign countries, document the importance of international collaborations and highlight the value for lessons learned to feed back to donor countries to improve their own programmes.

\section{Challenges for mutual learning}

Generally, the recognition of the strengths of the approaches and methodologies used by HASP has ensured an appetite for opportunities of mutual learning. However, this is not to suggest that challenges have not been encountered. The approaches and methodologies of HASP can be put in place without knowledge translation occurring. ${ }^{17} 18$ In fact, different organisations and individuals need to take ownership of the knowledge generated in order to ensure sensible and practical knowledge translation takes place. ${ }^{19}$ As to be expected, these organisations and individuals have different agendas and priorities and, at times, these must be negotiated to ensure the success of collaborative activities.

It is well documented that the use of new knowledge results from complex interactions between knowledge producers and knowledge users. ${ }^{20}$ One barrier to the use of new knowledge may be misconceptions on the part of both the knowledge producers and the knowledge users. These misconceptions may relate to the type of information necessary to develop interventions $^{21}$ or the objectives themselves. ${ }^{22}$ In addition, the acquisition and diffusion of knowledge is all too often a unidirectional process, which does not favour knowledge use. ${ }^{23} 24$

The political context in Pakistan had made progress difficult at times. For example, heightened political attention on injection drug use as a criminal activity made it difficult for government partners to adopt and act upon knowledge translation messages which employed a public health approach to harm reduction. Furthermore, the political nature of government appointments meant that there was little continuity in the HIV program staff, who are, ideally, important users of the knowledge generated through HASP.

The changing priorities of Canadian funding agencies also threaten the sustainability of projects. Although a second phase of HASP was planned, the Canadian International Development Agency reconsidered the scope of this investment and supported an 18-month extension instead to allow HASP to conduct a final round of surveillance, build additional capacity and hand its SGS model over to the Pakistani government. The government of Pakistan has been an essential HASP partner since its inception and now has the opportunity to ensure the survival and sustainability of the surveillance infrastructure that has been built.

\section{CONCLUSIONS}

There is a strong justification for Canada's investment in global public health. Over 8 years, a wealth of knowledge about implementing a SGS system in Pakistan has been generated and shared, contributing to the development of similar systems and guidelines elsewhere in Asia and the MENA region. The donor country, Canada, has also benefitted significantly. Methodologies for HIV surveillance that are currently being used in Canada were refined in Pakistan, and confidence and experience in these methodologies were built through HASP. Many of the collaborative relationships that have been developed between countries, agencies, and individuals will continue to exist and be fruitful for a diversity of intercountry initiatives and knowledge translation.

Despite the challenges, translating knowledge globally provides opportunities to build on each other's strengths and interests. As future projects are developed, it is essential that there is a shared vision of the objectives, trust, respect and an understanding of the needs of each partner. Through this process, the interaction between knowledge producers and knowledge users can be strengthened and thereby result in improved public health programmes.

Acknowledgements We thank the HASP surveillance team for their efforts in mapping, data collection, specimen collection, data entry and knowledge translation. We also thank the participants who took part in the surveys for their time and participation.

Contributors AA provided direction for the development of this manuscript's content, and provided critical reviews. LHT developed the first draft of the manuscript, coordinated reviews and revised later drafts. MM critically reviewed and revised drafts of the manuscript. JFB critically reviewed this manuscript and led the development of special supplement strategy.

Funding The Canada-Pakistan HIVIAIDS Surveillance Projected was funded by the Canadian International Development Agency. Contract number: CIDA PK 30849.

Competing interests None.

Ethics approval HOPE International in Pakistan and the Public Health Agency of Canada. 
Provenance and peer review Commissioned; externally peer reviewed. Contract number: CIDA PK 30849.

Open Access This is an Open Access article distributed in accordance with the Creative Commons Attribution Non Commercial (CC BY-NC 3.0) license, which permits others to distribute, remix, adapt, build upon this work non-commercially, and license their derivative works on different terms, provided the original work is properly cited and the use is non-commercial. See: http://creativecommons.org/ licenses/by-nc/3.0/

\section{REFERENCES}

1 World Health Organization (WHO). Second generation surveillance for HIV/AIDS. Geneval: WHO; 2011. Available from: http://www.who.int/hiv/topics/surveillance/ 2ndgen/en/ (accessed 4 Nov 2011).

2 Wilson D, Fraser N. Mixed HIV epidemic dynamics: epidemiology and program implications. Accra, Ghana: HIV Prevention in Mixed Epidemics Technical Consultation; 2011.

3 National AIDS Control Program (NACP). HIV second generation surveillance in Pakistan: national report round IV. Islamabad, Pakistan: NACP, 2012.

4 Canadian Institutes of Health Research (CIHR), 2009. About Knowledge Translation. Last modified May 8, 2009. Ottawa, Canada. http://www.cihr-irsc.gc.ca/e/29418. html (accessed 4 Nov 2011).

5 Evans, JR, Catillo, GT, Abed FA, et al. Commission on health research for development: health research: essential link to equity in development. Oxford: Oxford University Press, 1990.

6 Frenk J, Chen L. Overcoming gaps to advance global health equity: a symposium on new directions for research. Health Res Policy Syst 2011:9:11.

7 Pablos-Mendez A, Chunharas S, Lansang MA, et al. Knowledge translation in global health. Bull World Health Organ 2005:83:721-800.

8 Santesso N, Tugwell P. Knowledge translation in developing countries. I Contin Educ Health Prof 2006;26:87-96.

9 Pablos-Mendez A, Shademani R. Knowledge translation in global health. J Contin Educ Health Prof 2006;26:81-6.

10 Ramesh B, Washington R, Mondal S, et al. Sex work typology and risk for HIV in female sex workers (FSWs): findings from an integrated biological and behavioural assessment in the southern Indian state of Karnataka. 16th International AIDS Conference; 13-18 August 2006, Toronto, Canada.

11 World Health Organization (WHO). WHO manual for HIV drug resistance testing using dry blood spot specimens. Geneva: WHO; 2010.
12 Emmanuel F. Mapping of most at risk populations: An innovative approach to know "who" is at risk, "how many" are there and "where they are Regional Knowledge Hub for HIVIAIDS Surveillance Newsletter. Kerman, Iran, 2009:6.

13 World Health Organization (WHO). Regional Office for the Eastern Mediterranean HIV surveillance in the Middle East and North Africa: a handbook for surveillance planners and implementers. Nasr City, Cairo: WHO Regional Office for the Eastern Mediterranean, Joint United Nations Programme on HIVIAIDS; 2010

14 Public Health Agency of Canada (PHAC). I-track: enhanced surveillance of risk behaviours among people who inject drugs. Phase I report, 2006. Ottawa, Canada: Surveillance and Risk Assessment Division, Centre for Infectious Disease Prevention and Control, PHAC; 2006.

15 Public Health Agency of Canada (PHAC). E-SYS quick facts: chlamydia : a hidden epidemic in Canadian street youth. Surveillance and Risk Assessment Division, Centre for Infectious Disease Prevention and Control, PHAC; 2006.

16 Public Health Agency of Canada (PHAC). HIVIAIDS : M-Track. Ottawa, Canada: Surveillance and Risk Assessment Division, Centre for Infectious Disease Prevention and Control, PHAC; 2008. Available from: http://www.phac-aspc.gc.ca/aids-sida/ about/mtrack-eng.php (accessed 17 Dec 2011).

17 Webber D. Knowledge and policy. Int I Knowledge Transfer Utilization, 199192;4:6-35.

18 HEALNet Regionalization Research Centre. Using information in decisions (newsletter). 1998 Dec:1.

19 Lavis JN, Davies HTO, Oxman A, et al. Towards systematic reviews that inform health care management and policymaking. J Health Serv Res Policy 2005; 10(Suppl 1):35-48.

20 Weiss $\mathrm{CH}$. The many meanings of research utilisation. Public Adm Rev 1979;39:426-31.

21 Shrivastava P, Mitroff I.I. Enhancing organizational research utilization: the role of decision makers' assumptions. Acad Manag Rev 1984:9:18-26.

22 Rynes S L, Brown KG, Colbert AE. Research findings versus practitioner beliefs: seven common misconceptions about human resource practices. Acad Manag Exec 2002;16:92-102.

23 Westbrook J, Boethel M. General characteristics of effective dissemination and utilization (technical brief on the internet). Austin, Texas: National Center for the Dissemination of Disability Research; 1997. http://www.researchutilization.org/ matrix/resources/gcedu (accessed 17 Dec 2011).

24 Landry R, Amara N, Lamari M. Utilization of social science research knowledge in Canada. Res Policy 2001;30:333-49. 